\title{
Screening of Blood Donations for Zika Virus Infection - Puerto Rico, April 3-June 11, 2016
}

\begin{abstract}
Matthew J. Kuehnert ${ }^{1}$; Sridhar V. Basavaraju${ }^{1}$; Robin R. Moseley ${ }^{1}$; Lisa L. Pate ${ }^{2}$; Susan A. Galel ${ }^{2}$; Phillip C. Williamson ${ }^{3}$; Michael P. Busch ${ }^{4}$; Jose O. Alsina ${ }^{5}$; Consuelo Climent-Peris ${ }^{6}$; Peter W. Marks ${ }^{7}$; Jay S. Epstein ${ }^{7}$; Hira L. Nakhasi ${ }^{7}$; J. Peyton Hobson ${ }^{7}$; David A. Leiby ${ }^{7}$; Pradip N. Akolkar ${ }^{7}$; Lyle R. Petersen ${ }^{1}$; Brenda Rivera-Garcia ${ }^{8}$
\end{abstract}

On June 17, 2016, this report was posted as an MMWR Early Release on the MMWR website (http://www.cdc.gov/mmwr).

Transfusion-transmitted infections have been documented for several arboviruses, including West Nile and dengue viruses (1). Zika virus, a flavivirus transmitted primarily by Aedes aegypti mosquitoes that has been identified as a cause of congenital microcephaly and other serious brain defects (2), became recognized as a potential threat to blood safety after reports from a 2013-2014 outbreak in French Polynesia. Blood safety concerns were based on very high infection incidence in the population at large during epidemics, the high percentage of persons with asymptomatic infection, the high proportion of blood donations with evidence of Zika virus nucleic acid upon retrospective testing, and an estimated 7-10-day period of viremia (3). At least one instance of transfusion transmission of Zika virus has been documented in Brazil after the virus emerged there, likely in 2014 (4). Rapid epidemic spread spread has followed to other areas of the Americas, including Puerto Rico.

In February 2016, the Food and Drug Administration (FDA) issued recommendations for donor screening, donor deferral, and product management to reduce the risk for transfusiontransmitted Zika virus in the United States and its territories (5). In addition to behavioral- and health-risk questionnaires for blood donors in all areas, FDA recommends deferrals for donors in unaffected areas who recently lived in or visited an area with active mosquito-borne transmission of Zika virus. For establishments collecting blood in areas with active, local mosquito-borne transmission, such as Puerto Rico and other U.S. territories, the recommendations include discontinuing local blood collections and importing blood units from unaffected areas of the continental United States unless one of the following is implemented: 1) Zika virus screening of locally collected blood donations or 2) treatment of locally collected units with pathogen-reduction technology (FDA-approved only for plasma and apheresis platelets). In Puerto Rico, interventions initially were limited to importation of blood units from unaffected U.S. areas and to treatment of plasma and apheresis platelets with pathogen-reduction technology; no Zika virus screening test was available. On April 3, 2016, Zika virus screening of locally collected blood donations was implemented using a newly developed nucleic acid test (NAT) (cobas Zika, Roche Molecular Systems, Inc., Pleasanton, California) authorized by FDA under an investigational new drug application (IND) (G). As part of the IND, plasma samples from blood donors are screened individually, and specimens with reactive results are subjected to additional testing including an alternate NAT and immunoglobulin M serology. A blood donation with an initial reactive result by NAT is regarded as a presumptive viremic donor, indicating an infected donor, and is interdicted and removed from the blood supply.

During April 3-June 11, 2016, a total of 68 (0.5\%) presumptive viremic donors were identified from 12,777 donations tested. The highest weekly incidence was $1.1 \%$ for the latest week of reporting, June 5-June 11, and incidence has been increasing over time (Figure).

Although the blood donor population of Puerto Rico is not intended to be statistically representative of the general population, the increasing prevalence of $\mathrm{Zika}$ virus nucleic acid among blood donors likely reflects an overall increase in infection incidence in the population at large. Based on data from previous outbreaks caused by arboviruses transmitted by Aedes aegypti, the high incidence often associated with these outbreaks can result in a substantial proportion of the population becoming infected. For example, chikungunya virus was introduced into Puerto Rico in 2014. Retrospective screening for chikungunya virus nucleic acid was performed on blood donations collected during June-December 2014, and the estimated detectable viremia was $0.65 \%$, with a peak of $2.1 \%$ in October. Testing for chikungunya virus immunoglobulin $\mathrm{M}$ antibody of retained individual blood donation samples obtained during March 1-9, 2015, suggested that nearly 25\% of the Puerto Rico population became infected during the previous year's epidemic ( 7 ). Because viremia is only present days after acute infection, immunoglobulin $\mathrm{M}$ antibody can provide a more precise estimate of the burden of recent infection. The 2014-2015 chikungunya virus data suggest that detection of viremia in a relatively small proportion of blood donors each week can reflect a substantial proportion of the general population becoming infected during the course of an epidemic season.

Currently, no medication or vaccine is available to treat or prevent Zika virus disease. Prevention relies on avoidance of mosquito bites, elimination of mosquito breeding sites, community mosquito control, and taking measures to prevent sexual transmission. Screening of the U.S. blood supply using 
FIGURE. Proportion of screened blood donations reactive for Zika virus infection, by week of testing - Puerto Rico, April 3-June 11, 2016

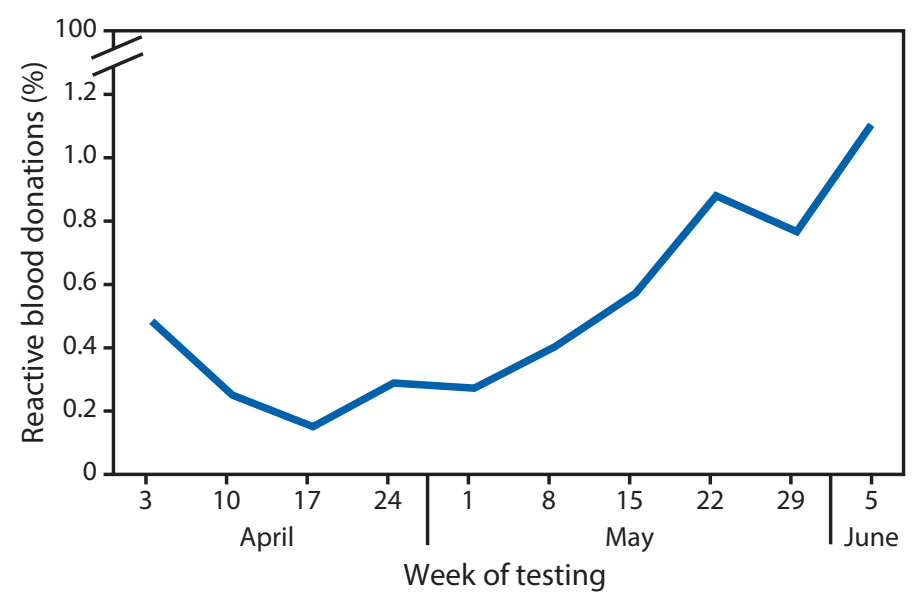

nucleic acid tests has markedly reduced the risk for transfusion transmission for multiple pathogens, including for West Nile virus after it was associated with arboviral epidemics in the United States. Measures to protect the blood supply from Zika virus, including donor deferrals, laboratory screening, and pathogen reduction technology, are expected to similarly reduce the risk for transfusion transmission.

\footnotetext{
${ }^{1}$ Zika virus response blood safety team, CDC; ${ }^{2}$ Roche Molecular Systems, Inc., Pleasanton, California; ${ }^{3}$ Creative Testing Solutions, Tempe, Arizona; ${ }^{4}$ Blood Systems Research Institute, San Francisco, California; ${ }^{5}$ Banco de Sangre de Servicios Mutuos, San Juan, Puerto Rico; ${ }^{6}$ Banco de Sangre de Puerto Rico; ${ }^{7}$ Food and Drug Administration, Silver Spring, Maryland; ${ }^{8}$ Puerto Rico Department of Health.
}

Corresponding author: Matthew J. Kuehnert, eocevent281@cdc.gov, 800-232-4636.

\section{References}

1. Petersen LR, Busch MP. Transfusion-transmitted arboviruses. Vox Sang 2010;98:495-503. http://dx.doi.org/10.1111/j.1423-0410.2009.01286.x

2. Rasmussen SA, Jamieson DJ, Honein MA, Petersen LR. Zika virus and birth defects—reviewing the evidence for causality. N Engl J Med 2016;374:1981-7. http://dx.doi.org/10.1056/NEJMsr1604338

3. Lanteri MC, Kleinman SH, Glynn SA, et al. Zika virus: a new threat to the safety of the blood supply with worldwide impact and implications. Transfusion 2016. Epub June 9, 2016. http://dx.doi.org/10.1111/ trf. 13677

4. Barjas-Castro ML, Angerami R, Cunha M, et al. Probable transfusiontransmitted Zika virus in Brazil. Transfusion 2016. In press.

5. Food and Drug Administration. Recommendations for donor screening, deferral, and product management to reduce the risk of transfusiontransmission of Zika virus. Silver Spring, MD: US Department of Health and Human Services, Food and Drug Administration; 2016. http://www.fda.gov/downloads/BiologicsBloodVaccines/ GuidanceComplianceRegulatoryInformation/Guidances/Blood/ UCM486360.pdf

6. Food and Drug Administration. FDA allows use of investigational test to screen blood donations for Zika virus. Silver Spring, MD: US Department of Health and Human Services, Food and Drug Administration; 2016. http://www.fda.gov/NewsEvents/Newsroom/PressAnnouncements/ ucm493081.htm

7. Simmons G, Brès V, Lu K, et al. High incidence of chikungunya virus frequency of viremic blood donations during epidemic, Puerto Rico, USA, 2014. Emerg Infect Dis 2016;22:1221-8. Epub April 2016. http:// dx.doi. org/10.3201/eid2207.160116 